\title{
EDITORIAL
}

\section{The elusive link between stenosis severity and prognosis in stable ischaemic heart disease}

\author{
F Crea, G A Lanza
}

Heart 2003;89:961-962

When assessing patients with stable ischaemic heart disease, cardiologists should not just take into account the number of critical stenoses detected at angiography, but should also consider patient symptoms, left ventricular function, the extent of myocardium at risk of ischaemia, and the extent of coronary atherosclerosis

t is well recognised that the number of coronary artery branches with a "critical stenosis" at angiography is a predictor of future cardiac events. ${ }^{1}$ Accordingly, the major scope of invasive cardiology is the identification and subsequent treatment of critical stenoses.

Yet a large body of evidence challenges the notion that critical stenoses are the most frequent site of future subtotal or total occlusions resulting in acute coronary syndromes. Indeed, serial angiographic studies have consistently shown that in about $85 \%$ of patients, coronary occlusion resulting in acute myocardial infarction occurs at the site of non-critical stenoses. ${ }^{2}$ Furthermore, severe stenoses often remain unchanged over time, whereas new critical obstructions may develop, even after a short follow up, in segments which had previously been found normal or near normal at angiography. ${ }^{3}$ Accordingly, crosssectional angiographic studies have shown that patients who present with stable angina as the first manifestation of ischaemic heart disease (IHD), compared to patients who present with an acute coronary syndrome, exhibit more severe coronary atherosclerosis, including a higher number of total coronary occlusions. ${ }^{5}$ Finally, it should be noted that even when total coronary occlusion occurs at the site of a chronic critical stenosis, it is likely to cause a small myocardial infarction or even remain clinically silent, owing to a well developed collateral circulation, and/or to ischaemic preconditioning.

\section{STENOSIS SEVERITY AND MAJOR CORONARY EVENTS}

Some studies have suggested that critical coronary stenoses with a complex morphology at angiography progress toward symptomatic occlusion more frequently than simple stenoses. Yet complex coronary stenoses may be associated with a worse outcome in patients with acute coronary syndromes, ${ }^{6}$ but their predictive value remains uncertain in patients with stable IHD. ${ }^{7}$

The reasons why acute coronary syndromes are typically caused by events occurring at the site of non-critical stenoses are now sufficiently clear.
Acute coronary events are caused by a complex interplay between local and systemic alterations resulting in thrombus formation and vasoconstriction, which are responsible for a sudden increase of coronary obstruction. Local thrombogenic and vasoconstrictor stimuli are not related to stenosis severity and may be enhanced by a concomitant systemic increase of platelet reactivity and of thrombin generation, by a systemic reduction of fibrinolytic activity, and by smooth muscle hyperreactivity, which are frequently observed in patients with acute coronary syndromes. Sudden activation of inflammatory cells within the atherosclerotic plaque appears to play a key role in the pathogenesis of acute coronary syndromes. ${ }^{8}$ The cytokines secreted by activated inflammatory cells have the potential to induce changes in endothelium function, transforming its anti-adhesive and anticoagulant properties into adhesive and procoagulant properties; furthermore, they may reduce matrix synthesis, and even increase its degradation, thus favouring plaque rupture. Finally, cytokines may stimulate endothelin-1 production which, in turn, causes vasoconstriction and enhances smooth muscle reactivity to other constrictors. ${ }^{8}$

Recent studies suggest that in patients with acute coronary syndromes inflammation is not limited to the culprit stenosis but is widespread in the coronary circulation. ${ }^{9}$ In acute coronary syndromes, a pronounced and persistent elevation of serum indexes of inflammation is associated with a worse outcome even in patients receiving the best current treatment, including percutaneous coronary intervention. ${ }^{10}$ Thus, it would appear that in high risk patients with unstable angina focal coronary interventions are needed, but they are insufficient to counteract destabilising inflammatory stimuli which diffusely and persistently affect coronary circulation. At the other extreme of the spectrum, in low risk patients with stable angina exhibiting preserved left ventricular function and effort tolerance, myocardial revascularisation is not superior to optimal medical treatment in the prevention of major coronary events, although it is more effective in symptom relief. ${ }^{11}$ In these patients aspirin and statin administration notably reduces the risk of major coronary events in the absence of clinically relevant effects on stenosis severity, thus highlighting that improvement of endothelial function, modulation of inflammatory cell reactivity, and reduction of platelet reactivity are probably more important goals than the mere mechanical treatment of stable critical stenoses. 


\section{CLINICAL ASSESSMENT OF STABLE ANGINA PATIENTS}

The article by Bigi and colleagues ${ }^{12}$ published in this issue of Heart lends further support to the notion that in patients with stable IHD the link between stenosis severity and major coronary events is rather elusive. These authors investigated the prognostic value of coronary angiography in a group of 228 consecutive patients with stable IHD. The authors compared the predictive value of the "vessel score" - that is, the number of epicardial vessels with critical stenoses-and of the "extent score", an angiographic index of coronary atherosclerotic burden. During an average follow up of 2.5 years, the authors observed nine deaths ( $1.6 \%$ per year), seven of which were cardiac ( $1.2 \%$ per year), and 32 non-fatal acute myocardial infarctions ( $12.8 \%$ per year), thus resulting in a total of 41 major coronary events (7.2\% per year). Although both extent score and vessel score predicted the outcome at the univariate analysis, extent score only predicted major coronary events at the multivariate analysis. Thus the atherosclerotic burden, rather than the number of critical stenoses, was the most predictive angiographic marker of risk. This contention was supported by the observation that clinical variables known to be strong predictors of major coronary events (for example, age and diabetes) showed a better correlation with the extent score than with the vessel score, a finding in keeping with the previous demonstration of a good correlation between extent score and atherogenic apolipoproteins. ${ }^{13}$

The study by Bigi and colleagues ${ }^{12}$ reminds us that in the assessment of patients with stable IHD cardiologists should not take into account the number of critical stenoses detected at angiography only. Indeed, the "cosmetic" effect of stenosis dilation does not necessarily translate into a better prognosis. In contrast, clinicians should take into account patient symptoms, left ventricular function, and extent of myocardium at risk of ischaemia. While myocardial revascularisation procedures are indicated in patients with three vessel disease, in particular in the presence of a depressed left ventricular function, the management of stable patients with one or two vessel disease is still controversial when symptoms are well controlled by medical treatment. In this subset of patients the extent score might help to identify patients with a worse prognosis who might benefit from a more aggressive risk factor control or, possibly, from myocardial revascularisation.
Unfortunately, Bigi and colleagues ${ }^{12}$ do not establish in their study whether the prognostic information provided by the extent score is additive to that given by the non-invasive assessment of myocardium at risk of ischaemia or by biomarkers such as $\mathrm{C}$ reactive protein, thus making its incorporation into currently accepted risk algorithms rather problematic.

Authors' affiliations

F Crea, G A Lanza, Istituto di Cardiologia, Università Cattolica del Sacro Cuore, Roma, Italy

\section{REFERENCES}

1 Humphries JO, Kuller L, Ross R, et al. Natural history of ischemic heart disease in relation to arteriographic findings. Circulation 1974:49:489-97.

2 Falk E, Shah PK, Fuster V. Coronary plaque disruption. Circulation 1995;92:657-71.

3 Kaski JC, Chester MR, Chen L, et al. Rapid angiographic progression of coronary artery disease in patients with angina pectoris. The role of complex stenosis morphology. Circulation 1995;92:2058-65

4 Yokoya K, Takatsu H, Suzuki T, et al. Process of progression of coronary artery lesions from mild or moderate stenosis to moderate or severe stenosis. A study based on four serial coronary arteriograms per year. Circulation 1999:100:903-9.

5 Cianflone D, Ciccirillo F, Buffon A, et al. Comparison of coronary angiographic narrowing in stable angina pectoris, unstable angina pectoris, and in acute myocardial infarction. Am J Cardiol 1995;76:215-9.

6 Davies SW, Marchant B, Lyons JP, et al. Irregular coronary lesion morphology after thrombolysis predicts early clinical instability. J Am Coll Cardiol 1991;18:669-74.

7 Ambrose JA. Coronary arteriographic analysis and angiographic morphology. J Am Coll Cardiol 1989;13:1492-4.

8 Libby $\mathbf{P}$, Ridker PM Maseri A. Inflammation and atherosclerosis. Circulation 2002; 105: $1135-43$

9 Buffon A, Biasucci LM, Liuzzo G, et al. Widespread coronary inflammation in unstable angina. N Engl J Med 2002;347:5-12.

10 Mueller C, Buettner HJ, Hodgson JM, et al. Inflammation and long-term mortality after non-ST elevation acute coronary syndrome treated with a very early invasive strategy in 1042 consecutive patients. Circulation 2002; 105: 1412-5.

11 Pitt B, Waters D, Brown WV, et al, for the Atorvastatin Versus Revascularization Treatment (AVERT) Investigators. Aggressive lipid-lowering therapy compared with angioplasty in stable coronary artery disease. N Engl J Med 1999;341:70-6.

12 Bigi R, Cortigiani L, Colombo P, et al. Prognostic and clinical correlates of angiographically diffuse non-obstructive coronary lesions. Heart 2003:89:1009-13.

13 Sullivan DR, Marwick TH, Freedman SB. A new method of scoring coronary angiograms to reflect extent of coronary atherosclerosis and improve correlation with major risk factors. Am Heart J 1990;1 19:1262-7. 